\title{
Differently Expressed 'Early' Flavonoid Synthesis Genes in Wheat Seedlings Become to Be Co-regulated under Salinity Stress
}

\author{
O.Y. Shoeva ${ }^{1 *}$ and E.K. KhlESTKINA ${ }^{1,2}$ \\ ${ }^{1}$ Institute of Cytology and Genetics, Siberian Branch of the Russian Academy of Sciences, \\ Lavrentjeva Ave. 10, Novosibirsk, 630090 Russia \\ ${ }^{2}$ Novosibirsk State University, Pirogova St. 2, Novosibirsk, 630090 Russia \\ (Received 4 September 2014; Accepted 24 March 2015; \\ Communicated by $\mathrm{H}$. Grausgruber)
}

\begin{abstract}
Synthesis of flavonoid compounds in plants is associated with their response to environmental stress; however, the way in which the transcription of the relevant structural genes is regulated in stressed plants is still obscure. Transcription of the 'early' flavonoid synthesis genes Chi-1 and F3h-1 in the wheat coleoptile was investigated by quantitative real-time PCR in seedlings exposed to $100 \mathrm{mM}$ or $200 \mathrm{mM} \mathrm{NaCl}$. Under mild stress, transcript abundance of both Chi-1 and F3h-1 was increased significantly after six days of exposure. Under severe stress, the level of transcription was the same or even lower than that seen in nonstressed seedlings. In non-stressed conditions, the transcription patterns of Chi-1 and F3h-1 were quite distinct from one another, whereas under stress they became similar. An observed alteration in structural genes regulation mode under stress conditions may optimize flavonoid biosynthesis pathway to produce protective compounds with maximum efficiency.
\end{abstract}

Keywords: salinity tolerance, Triticum aestivum L., transcript abundance, qRT-PCR

\section{Introduction}

The flavonoids are a group of phenolic plant secondary metabolites which make an important contribution to the response to both biotic and abioitic stress (Treutter 2006; Khlestkina 2013). In bread wheat (Triticum aestivum), the synthesis of flavonoids can be induced by e.g. Hessian fly infestation, salinity, drought, and low temperature stress (Giovanini et al. 2006; Olenichenko et al. 2006, 2008; Tereshchenko et al. 2012; Ma et al. 2014). A comparison between near-isogenic wheat lines has suggested a correlation between drought tolerance and the intensity of pigmentation of the coleoptile and pericarp produced by anthocyanins (Tereshchenko et al. 2012). Although the molecular basis of anthocyanin synthesis has been thoroughly investigated (Winkel-Shirley 2001), little is known concerning the regulation under stress of the relevant structural genes. In wheat, the regulation of the gene encoding flavanone 3-hydroxylase $(\mathrm{F} 3 \mathrm{H})$ is of particular inter-

*Corresponding author; E-mail: olesya_ter@bionet.nsc.ru; Phone: +7(383)363-49-52; Fax: +7(383)333-12-78 
est, since $\mathrm{F} 3 \mathrm{H}$ activation is the major regulatory checkpoint in anthocyanin synthesis (Khlestkina et al. 2008, 2010; Tereshchenko et al. 2013).

Three $F 3 h$ homoeologs and one paralog are present in the bread wheat genome (Khlestkina et al. 2008, 2013; Himi et al. 2011). The F3h-1 homoeologs are all involved in anthocyanin synthesis (Shoeva and Khlestkina 2013). The products of $F 3 h-1$, along with those of $C h i$ (chalcone-flavanone isomerase) and $C h s$ (chalcone synthase) are all involved in the early stage of the anthocyanin synthesis pathway; the genes are co-regulated in some plant species (Martin et al. 1991; Quattrocchio et al. 1993), but not so in wheat (Tereshchenko et al. 2013). Chalcone-flavanone isomerases are responsible for the formation of nine of the twelve major flavonoid subgroups (Winkel-Shirley 2001). The wheat genome harbors three homoeologous copies of Chi-1, all of which are transcriptionally active in both pigmented and non-pigmented tissue (Shoeva et al. 2014). The present study set out to expose the temporal transcriptional behavior Chi-1 and F3h-1 in the wheat coleoptile in response to imposed salinity stress.

\section{Materials and Methods}

\section{Plant material and RNA extraction}

Total RNA was extracted from seedlings of the bread wheat cultivar 'Saratovskaya 29', which has pronounced anthocyanin pigmentation in its coleoptile (Khlestkina et al. 2010). Seeds were germinated on moist filter paper at $20{ }^{\circ} \mathrm{C}$ under a $12 \mathrm{~h}$ photoperiod, and after one day were exposed to 0 (control), $100 \mathrm{mM}$ or $200 \mathrm{mM} \mathrm{NaCl}$. Three replicates of each treatment were imposed, with each replicate consisting of 16 seedlings. Four seedlings per replicate were pooled every $24 \mathrm{~h}$ between days 3 and 6 for subsequent RNA extraction by a QIAGEN Plant Rneasy Kit (www.qiagen.com), followed by a DNAse treatment. Concentration of RNA was measured by spectrophotometer (SmartSpec ${ }^{\mathrm{TM}}$ Plus, BioRad).

\section{Quantitative real-time PCR ( $q R T-P C R)$}

Single-stranded cDNA was synthesized from $1 \mu \mathrm{g}$ total RNA using a $(\mathrm{dT})_{15}$ primer and the QIAGEN Omniscript Reverse Transcription kit in a $20 \mu \mathrm{l}$ reaction. The subsequent qRT-PCRs were formulated with a QIAGEN QuantiTect SYBR Green kit and run on the 7900 HT Fast Real-Time PCR System (Applied Biosystems, USA). The primer pairs for amplifying F3h-1 were homoeolog-specific (Khlestkina et al. 2008), while all three Chi-1 homoelogs were amplified together using the pair 5'-CGGGGGCAAGTTCATCAAGT-3' and 5'-CAGCGGCAGGATCATCGTCA-3'. Known quantities of cloned cDNA product were used to calibrate a standard curve. Each sample was represented by three technical replicates. Transcript abundances were normalized with respect to those obtained for TaAct (AY663392), based on amplicons generated by the primer pair 5'-CTGACGGTGAGGACATC-3' and 5'-CCCATCCCTACCATGAC-3'. The primers were designed using OLIGO software (Offerman and Rychlik 2003). 


\section{Statistical analysis}

Data are presented as means \pm standard error (SE). Spearman rank correlation between the genes' expression levels was carried out using Statistica v6.1 software package (StatSoft Inc., USA). Differences between transcript abundances in non-stressed seedlings and those exposed to either $100 \mathrm{mM}$ or $200 \mathrm{mM} \mathrm{NaCl}$ were tested by Dunnett's post hoc test, taking $p \leq 0.05$ as the significance threshold.

\section{Results}

\section{Chi-1 transcription}

The transcription of $\mathrm{Chi}-\mathrm{I}$ in seedlings exposed to salinity stress for up to six days is illustrated in Fig. 1a. In three day old seedlings, the level of Chi-1 transcript was suppressed by the salinity stress imposed by both concentrations of $\mathrm{NaCl}$. However, by day 4 , it had recovered to the control level in the seedlings exposed to $100 \mathrm{mM} \mathrm{NaCl}$, while in the more severely stressed seedlings, transcription remained lower than in the control. Transcription was down-regulated in the seedlings exposed to $200 \mathrm{mM} \mathrm{NaCl}$ on day 5, but was up-regulated in those exposed to $100 \mathrm{mM} \mathrm{NaCl}$ on day 6 . It can be concluded that salinity affects expression of the Chi-1 gene, but this effect is different under distinct $\mathrm{NaCl}$ concentrations.
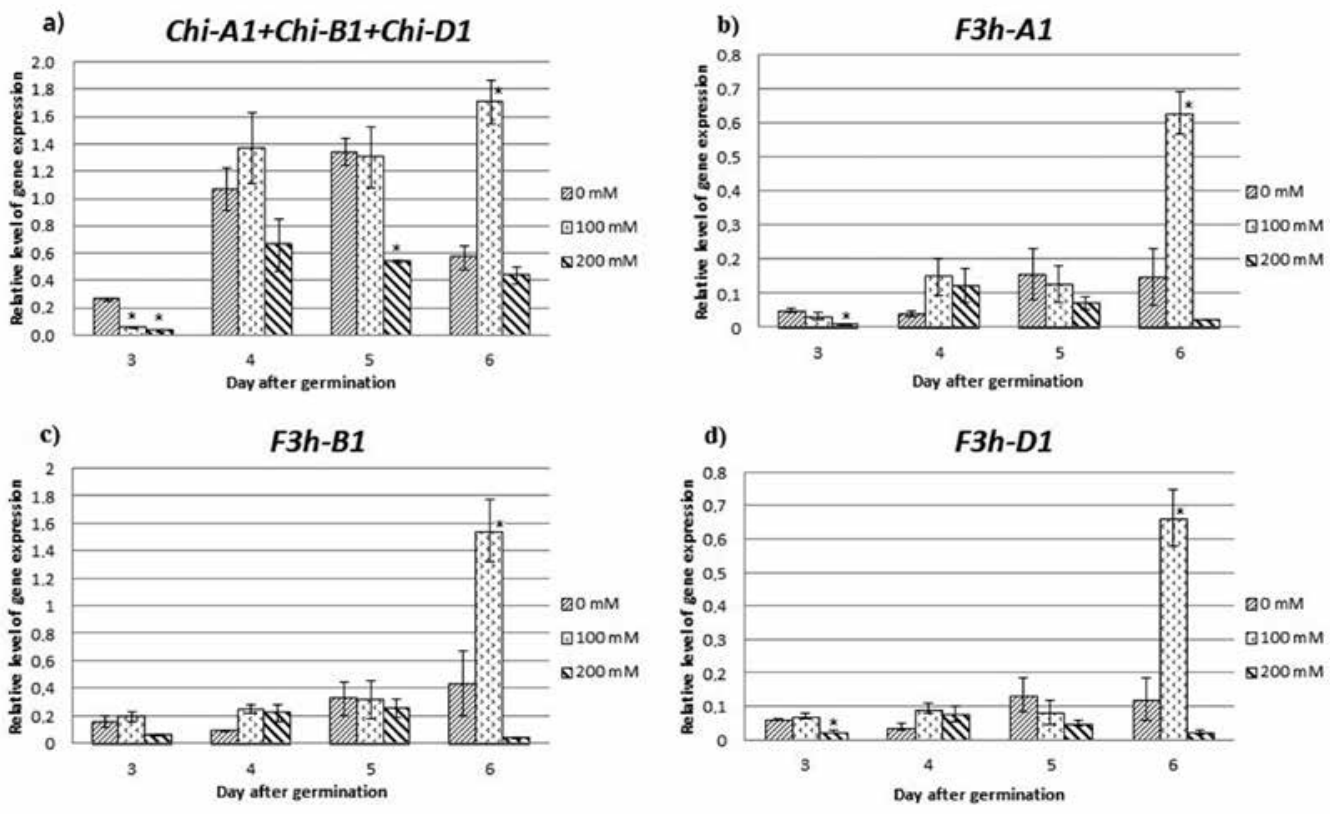

Figure 1. Transcription profiles (a) $C h i-1$, (b) $F 3 h-A 1$, (c) $F 3 h-B 1$ and (d) $F 3 h-D 1$ under control $(0 \mathrm{mM} \mathrm{NaCl})$ and salinity stressed conditions $(100$ and $200 \mathrm{mM} \mathrm{NaCl}) .{ }^{*}$ - differences statistically significant at $p \leq 0.05$ 


\section{F3h-1 transcription}

Transcription of each of the three individual $F 3 h-1$ homoeologs was suppressed by the presence of $200 \mathrm{mM} \mathrm{NaCl}$ on day 3, but induced markedly by the presence of $100 \mathrm{mM}$ $\mathrm{NaCl}$ on day 6 (Fig. $1 \mathrm{~b}-\mathrm{d}$ ). The transcript levels of the three genes were well correlated with one another throughout, always remaining at or above 0.69 (Table 1). As for Chi-1, salinity stress affects expression of the $F 3 h-1$ gene but this effect is different under distinct $\mathrm{NaCl}$ concentrations.

\section{Correlation between F3h-1 and Chi-1 transcription}

The abundance of $C h i-1$ and $F 3 h-1$ transcript was not correlated in the absence of salinity stress, but in its presence (both at $100 \mathrm{mM}$ and $200 \mathrm{mM} \mathrm{NaCl}$ ), there was a strong correlation (Table 1). It can be concluded that in optimal growing conditions some differences in regulation mode of $F 3 h-1$ and $C h i-1$ exist, whereas under salinity conditions the 'early' flavonoid biosynthesis genes are co-regulated (Table 1).

Table 1. Spearman's correlation between the transcript abundance of Chi-I and F3h-1

\begin{tabular}{|c|c|c|c|}
\hline $0 \mathrm{mM}$ & $F 3 h-B 1$ & $F 3 h-D 1$ & $C h i-1$ \\
\hline$F 3 h-A 1$ & $0.84^{*}$ & $0.87^{*}$ & 0.35 \\
\hline$F 3 h-B 1$ & & $0.91^{*}$ & 0.25 \\
\hline$F 3 h-D 1$ & & & 0.13 \\
\hline $100 \mathrm{mM}$ & F3h-B1 & F3h-D1 & Chi-1 \\
\hline$F 3 h-A 1$ & $0.93^{*}$ & $0.93^{*}$ & $0.78^{*}$ \\
\hline$F 3 h-B 1$ & & $0.99^{*}$ & $0.74^{*}$ \\
\hline$F 3 h-D 1$ & & & $0.78^{*}$ \\
\hline $200 \mathrm{mM}$ & F3h-B1 & F3h-D1 & Chi-1 \\
\hline$F 3 h-A 1$ & $0.69^{*}$ & $0.83^{*}$ & $0.96^{*}$ \\
\hline$F 3 h-B 1$ & & $0.93^{*}$ & $0.58^{*}$ \\
\hline$F 3 h-D 1$ & & & $0.76^{*}$ \\
\hline
\end{tabular}

* Statistically significant at $p \leq 0.05$.

\section{Discussion}

Intensification of the flavonoid synthesis and expression of the related genes under stress conditions was widely reported in diverse plant species (Ithal and Reddy 2004; Lo Piero et al. 2005; Walia et al. 2005; Ma et al. 2014). The accumulation of flavonoids and expression of those genes in response to stress are reported to be genotype dependent. For instance, saline treatment increased the accumulation of total anthocyanins in fruits of saltsensitive tomato cultivar 'Sun Black' (2-fold increase), while it reduced it in fruits of 
salt-tolerant cultivar 'Anthocyanin fruit type' (10-fold decrease) (Borghesi et al. 2011). In rice, flavonoid pathway genes were significantly induced by salinity in salt-sensitive IR29 genotype, but none of the genes were induced at a statistically significant level in stressed salt-tolerance sample FL478 (Walia et al. 2005).

According to the current study expression of the $C h i$ and $F 3 h$ genes in coleoptile of wheat cultivar 'Saratovskaya 29' is belatedly activated and only under milder stress regime (Fig. 1). Differences in the timing of induction of stress-activated genes are normally explained by variation in which proteins or signaling molecules they respond to (Hirayama and Shinozaki 2010).

Under normal growing conditions, the structural genes responsible for anthocyanin synthesis are regulated by a MYB/MYC/WD40 complex, which is responsive to a complex combination of both environmental (light, temperature) and biochemical (sugars and hormones) factors (Das et al. 2012). In Medicago truncatula, Shen et al. (2010) showed that there was a $12 \mathrm{~h}$ delay between the up-regulation of $F 3 h$ in the root and stem in response to salinity stress, whereas no induction was noted in the leaf even $48 \mathrm{~h}$ after the stress treatment had been imposed. The suggestion was that this delay reflected inefficiencies in the transport of key secondary metabolite signaling molecules. Late activation (on the fifth day of exposure to salinity stress) of a Chi gene has also been observed in the shoot of the Antarctic extremophile Deschampsia antarctica (Zamora et al. 2013).

The present experiments have demonstrated that the 'early' flavonoid synthesis genes F3h-1 and Chi-1 are not rapidly up-regulated in the wheat seedling and are sensitive to an intermediate level of salinity stress, whereas under high salinity stress, their transcription is the same or even lower than that seen in non-stressed seedlings. This allows suggesting that flavonoid biosynthesis can be suppressed under severe stress conditions and hence flavonoids in some cases cannot help plants overcome intensive stress.

The data show that the way in which $F 3 h-1$ and $C h i-1$ is regulated is dependent on the growing conditions experienced by the plant: under optimal growing conditions, the two genes are regulated independently, while under salinity stress conditions, they appear to be co-regulated (Table 1). F3h-1 transcript abundance is strongly correlated with the presence of pigmentation, while that of Chi-l is not (Khlestkina et al. 2008, 2010; Tereshchenko et al. 2013; Shoeva et al. 2014). The independent regulation of various flavonoid synthesis structural genes under optimal growing conditions has been widely reported (Martin et al. 1991; Quattrocchio et al. 1993; Tereshchenko et al. 2013).

Under low temperature conditions, the transcription patterns of genes encoding chalcone synthase (Chs), dihydroflavonol 4-reductase (Dfr), UDP-glucose:flavonoid 3-Oglucosyltransferase (Ufgt) and glutathione S-transferase (Gst) in the maize seedling are distinct (Christie et al. 1994). Similarly, in red colored oranges, the transcription of Chs, $D f r$ and Ufgt differing from Pal (phenylalanine ammonia-lyase) is affected by brief low temperature stress (Lo Piero et al. 2005). In rice, the transcription of Pal and Chs is not induced by salinity stress, although that of $D f r$ and Ans (anthocyanidin synthase) is (Ithal and Reddy 2004).

The distinct regulation of genes active in a single metabolic pathway can result in a diversity of flavonoid content, both qualitatively and quantitatively. Additional stress- 
specific transcriptional factors can be involved in regulation of flavonoid synthesis genes expression. For instance, in Arabidopsis, bZIP transcriptional regulator AIR1 has been assumed to be involved in the regulation of various steps of the flavonoid pathway and itself regulated by the salt-stress response signalling machinery (Van Oosten et al. 2013).

A stress-imposed alteration in the mode of regulation of this class of structural gene can be used by the plant to optimize the activity of a given metabolic pathway, allowing protective compounds to be generated with maximum efficiency.

\section{Acknowledgements}

We thank Dr Robert Koebner (www.smartenglish.co.uk) for linguistic assistance during the preparation of the manuscript. This study was partially supported by RFBR (grant No 14-04-31637), a grant from the President of the Russian Federation (MD-2615.2013.4), and the State Budget Programme (Project No VI.53.1.5.).

\section{References}

Borghesi, E., González-Miret, M.L., Escudero-Gilete, M.L., Malorgio, F., Heredia, F.J., Meléndez-Martínez, A.J. 2011. Effects of salinity stress on carotenoids, anthocyanins, and color of diverse tomato genotypes. J. Agric. Food Chem. 59:11676-11682.

Christie, P.J., Alfenito, M.R., Walbot, V. 1994. Impact of low-temperature stress on general phenylpropanoid and anthocyanin pathways - enhancement of transcript abundance and anthocyanin pigmentation in maize seedlings. Planta 194:541-549.

Das, P.K., Shin, D.H., Choi, S.-B., Park, Y.-I. 2012. Sugar-hormone cross-talk in anthocyanin biosynthesis. Mol. Cells 34:501-507.

Giovanini, M.P., Puthoff, D.P., Nemacheck, J.A., Mittapalli, O., Saltzmann, K.D., Ohm, H.W., Shukle, R.H., Williams C.E. 2006. Gene-for-gene defense of wheat against the Hessian fly lacks a classical oxidative burst. Mol. Plant-Microbe Interact. 19:1023-1033.

Himi, E., Maekawa, M., Noda, K. 2011. Differential expression of three flavanone 3-hydroxylase (F3H) genes in grains and coleoptiles of wheat. Int. J. Plant Genomic ID:369460.

Hirayama, T., Shinozaki K. 2010. Research on plant abiotic stress responses in the post-genome era: past, present and future. Plant J. 61:1041-1052.

Ithal, N., Reddy, A.R. 2004. Rice flavonoid pathway genes, OsDfr and OsAns, are induced by dehydration, high salt and ABA, and contain stress responsive promoter elements that interact with the transcription activator, OsC1-MYB. Plant Sci. 166:1505-1513.

Khlestkina, E.K. 2013. The adaptive role of flavonoids: emphasis on cereals. Cereal Res. Commun. 41:185198.

Khlestkina, E.K., Dobrovolskaya, O.B., Leonova, I.N., Salina, E.A. 2013. Diversification of the duplicated $F 3 h$ genes in Triticeae. J. Mol. Evol. 76:261-266.

Khlestkina, E.K., Röder, M.S., Salina, E.A. 2008. Relationship between homoeologous regulatory and structural genes in allopolyploid genome - a case study in bread wheat. BMC Plant Biol. 8:88.

Khlestkina, E.K., Röder, M.S., Pshenichnikova, T.A., Börner, A. 2010. Functional diversity at the $R c$ (red coleoptile) gene in bread wheat. Mol. Breeding 25:125-132.

Lo Piero, A.R., Puglisi, I., Rapisarda, P., Petrone, G. 2005. Anthocyanins accumulation and related gene expression in red orange fruit induced by low temperature storage. J. Agric. Food Chem. 53:9083-9088.

Ma, D., Sun, D., Wang, C., Li, Y., Guo, T. 2014. Expression of flavonoid biosynthesis genes and accumulation of flavonoid in wheat leaves in response to drought stress. Plant Physiol. Biochem. 80:60-66. 
Martin, C., Prescott, A., MacKay, S., Bartlett, J., Vrijlandt, E. 1991. Control of anthocyanin biosynthesis in flowers of Antirrhinum majus. Plant J. 1:37-49.

Offerman, J.D., Rychlik, W. 2003. Oligo primer analysis software. In: Krawetz, S.A., Womble D.D. (eds), Introduction to Bioinformatics: a Theoretical and Practical Approach. Humana Press. New Jersey, USA. pp. 345-361.

Olenichenko, N.A., Ossipov, V.I., Zagoskina, N.V. 2006. Effect of cold hardening on the phenolic complex of winter wheat leaves. Russ. J. Plant Physiol. 53:495-500.

Olenichenko, N.A., Zagoskina, N.V., Astakhova, N.V., Trunova, T.I., Kuznetsov, Yu.V. 2008. Primary and secondary metabolism of winter wheat under cold hardening and treatment with antioxidants. Appl. Biochem. Microbiol. 44:535-540.

Quattrocchio, F., Wing, J.F., Leppen, H.T.C., Mol, J.N.M., Koes R.E. 1993. Regulatory genes controlling anthocyanin pigmentation are functionally conserved among plant species and have distinct sets of target genes. Plant Cell 5:1497-1512.

Shen, X. Y., Martens, S., Chen, M.L., Li, D.F., Dong, J.L., Wang, T. 2010. Cloning and characterization of a functional flavanone-3 beta-hydroxylase gene from Medicago truncatula. Mol. Biol. Rep. 37:3283-3289.

Shoeva, O.Y., Khlestkina, E.K. 2013. F3h gene expression in various organs of wheat. Mol. Biol. 47:901-903.

Shoeva, O.Y., Khlestkina, E.K., Berges, H., Salina, E.A. 2014. The homoeologous genes encoding chalconeflavanone isomerase in Triticum aestivum L.: structural characterization and expression in different parts of wheat plant. Gene 538:334-341.

Tereshchenko, O.Y., Arbuzova, V.S., Khlestkina, E.K. 2013. Allelic state of the genes conferring purple pigmentation in different wheat organs predetermines transcriptional activity of the anthocyanin biosynthesis structural genes. J. Cereal Sci. 57:10-13.

Tereshchenko, O.Y., Khlestkina, E.K., Gordeeva, E.I., Arbuzova, V.S., Salina, E.A. 2012. Relationship between anthocyanin biosynthesis and abiotic stress in wheat. In: Börner, A., Kobijlski, B. (eds), Proceedings of the 15th International EWAC Conference, 2011, Novi Sad, Serbia. pp. 72-75.

Treutter, D. 2006. Significance of flavonoids in plant resistance: a review. Environ. Chem. Lett. 4:147-157.

Van Oosten, M.J., Sharkhuu, A., Batelli, G., Bressan, R.A., Maggio, A. 2013. The Arabidopsis thaliana mutant airl implicates SOS3 in the regulation of anthocyanins under salt stress. Plant Mol. Biol. 83:405-415.

Walia, H., Wilson, C., Condamine, P., Liu, X., Ismail, A.M., Zeng, L., Wanamaker, S.I., Mandal, J., Xu, J., Cui, X.P., Close, T.J. 2005. Comparative transcriptional profiling of two contrasting rice genotypes under salinity stress during the vegetative growth stage. Plant Physiol. 139:822-835.

Winkel-Shirley, B. 2001. It takes garden. How work on diverse plant species has contributed to an understanding of flavonoid metabolism. Plant Physiol. 127:1399-1404.

Zamora, P., Pardo, A., Fierro, A., Prieto, H., Zuniga, G.E. 2013. Molecular characterization of the chalcone isomerase gene family in Deschampsia antarctica. Polar. Biol. 36:1269-1280. 